\title{
EXISTENCE RESULTS ON POSITIVE PERIODIC SOLUTIONS FOR IMPULSIVE FUNCTIONAL DIFFERENTIAL EQUATIONS
}

\author{
YuJi LiU \\ Guangdong University of Business Studies, P. R. China
}

\begin{abstract}
A class of first order nonlinear functional differential equations with impulses is studied. It is shown that there exist one or two positive $T$-periodic solutions under certain assumptions, and no positive $T$-periodic solution under some other assumptions. Applications to some impulsive biological models and an example, which can not be covered by known results, are given to illustrate the main results.
\end{abstract}

\section{INTRODUCTION}

The theory of impulsive functional differential equations (IFDE for short) is an active area of research, see the papers [1,3,4,9-27,29,32,33,37,38,40-42]. For some general mathematical aspects of IFDE we refer to the text book [8].

In known papers $[2,11,14-16,20,21,29,34,37,39-41]$, the existence of positive periodic solutions for the IFDEs of the form

(1.1)

$$
\left\{\begin{array}{l}
x^{\prime}(t)=-a(t) x(t)+\lambda h(t) f(t, x(t-\tau(t, x(t)))), t \in \mathbb{R}, t \neq t_{k}, k \in \mathbb{Z} \\
x\left(t_{k}^{+}\right)=\left(1+b_{k}\right) x\left(t_{k}\right), k \in \mathbb{Z}
\end{array}\right.
$$

and

$$
\left\{\begin{array}{l}
x^{\prime}(t)=a(t) x(t)-\lambda h(t) f(t, x(t-\delta(t))), t \in \mathbb{R}, t \neq t_{k}, k \in \mathbb{Z} \\
x\left(t_{k}^{+}\right)=\left(1+b_{k}\right) x\left(t_{k}\right), k \in \mathbb{Z}
\end{array}\right.
$$

2010 Mathematics Subject Classification. 34B10, 34B15, 34K15, 34K10, 34C25, 92D25.

Key words and phrases. Impulse, first order functional differential equation, impulsive biological model, positive $T$-periodic solution, fixed point theorem.

The author is supported by Natural Science Foundation of Hunan province, P. R. China (No:06JJ5008) and Natural Science Foundation of Guangdong province (No:7004569). 
were studied, where $b_{k}>-1$ for all $k \in Z, \lambda>0, a, h, \delta$ are positive $T$ periodic functions, $f(t, x)$ and $\tau(t, x)$ are nonnegative and $T$-periodic in $t$ and continuous in $x$.

In $[5-8,12,13,17,22,28,30,31,33,35,36]$, the existence of positive periodic solutions of the following IFDEs

$$
\begin{gathered}
\left\{\begin{array}{l}
N^{\prime}(t)=-\mu N(t)+\lambda p e^{-r N(t-\tau)}, t \neq t_{k}, k \in \mathbb{Z}, \\
N\left(t_{k}\right)=\left(1+b_{k}\right) N\left(t_{k}^{-}\right), k \in \mathbb{Z},
\end{array}\right. \\
\left\{\begin{array}{l}
N^{\prime}(t)=-\mu N(t)+\lambda p \frac{N^{n}(t-\tau)}{1+r N^{m}(t-\tau)}, t \neq t_{k}, k \in \mathbb{Z}, \\
N\left(t_{k}\right)=\left(1+b_{k}\right) N\left(t_{k}^{-}\right), k \in \mathbb{Z},
\end{array}\right.
\end{gathered}
$$

and

$$
\left\{\begin{array}{l}
N^{\prime}(t)=-\mu N(t)+\lambda p N(t-\tau) e^{-r N(t-\tau)}, t \neq t_{k}, k \in \mathbb{Z} \\
N\left(t_{k}\right)=\left(1+b_{k}\right) N\left(t_{k}^{-}\right), k \in \mathbb{Z}
\end{array}\right.
$$

were studied, where $\mu$ is the probability of death of the biological population, $p$ and $r$ are positive constants related to the production of the biological population per unit time and $\tau$ is the time required to produce a new biological population, $m>0, n \geq 0$ real numbers and $\lambda>0$ a parameter and $b_{k}>-1$ for all $k \in \mathbb{Z}, N(t)$ denotes the number of the biological population at time $t$.

System (1.3), (1.4) and (1.5) are called impulsive model of red blood cell system, impulsive hematopoiesis model and impulsive Nicholson's Blowfly model, respectively.

In the case when $\mu$, the probability of death of the biological population, depends on the time and the total population number $N(t)$ at time $t$, one should replace $\mu$ by $\mu(t) \phi(N(t))$. So it is interesting to consider the existence of positive periodic solutions of the following impulsive models

$$
\begin{gathered}
\left\{\begin{array}{l}
N^{\prime}(t)=-\mu(t) \phi(N(t)) N(t)+\lambda p(t) e^{-r(t) N(t-\tau(t))}, t \neq t_{k}, k \in \mathbb{Z}, \\
N\left(t_{k}\right)=\left(1+b_{k}\right) N\left(t_{k}^{-}\right), k \in \mathbb{Z},
\end{array}\right. \\
\left\{\begin{array}{l}
N^{\prime}(t)=-\mu(t) \phi(N(t)) N(t)+\lambda p(t) \frac{N^{n}(t-\tau(t))}{1+r(t) N^{m}(t-\tau(t))}, t \neq t_{k}, k \in \mathbb{Z}, \\
N\left(t_{k}\right)=\left(1+b_{k}\right) N\left(t_{k}^{-}\right), k \in \mathbb{Z},
\end{array}\right.
\end{gathered}
$$

and

$$
\left\{\begin{array}{l}
N^{\prime}(t)=-\mu(t) \phi(N(t)) N(t)+\lambda p(t) N(t-\tau) e^{-r(t) N(t-\tau(t))}, t \neq t_{k}, k \in \mathbb{Z} \\
N\left(t_{k}\right)=\left(1+b_{k}\right) N\left(t_{k}^{-}\right), k \in \mathbb{Z},
\end{array}\right.
$$

where $b_{k}>-1$ for all $k \in \mathbb{Z}, \lambda>0$ a parameter, $\phi:[0,+\infty) \rightarrow[0,+\infty)$ is continuous and satisfies that there exist positive numbers $l_{1}<l_{2}$ such that $l_{1} \leq \phi(x) \leq l_{2}$ for all $x \in[0,+\infty), \mu, p, r$ and $\tau$ are positive $T$-periodic functions, $t_{k}$ are positive real number sequence with $\lim _{k \rightarrow+\infty} t_{k}=+\infty$, $b_{k}>-1$ constants for all $k \in \mathbb{Z}$ with $\prod_{t<t_{k}<t+T}\left(1+b_{k}\right)=$ constant for all $t \in \mathbb{R}$. 
Motivated by this reson, we consider the following more general first order functional differential equation

$$
\begin{aligned}
x^{\prime}(t)= & -a(t) \phi(x(t)) x(t)+\lambda h(t) f(t, x(t-\tau(t, x(t)))), \\
& t \in \mathbb{R}, t \neq t_{k}, k \in \mathbb{Z},
\end{aligned}
$$

with the impulse effects

$$
x\left(t_{k}\right)=\left(1+b_{k}\right) x\left(t_{k}^{-}\right), k \in \mathbb{Z},
$$

where

$* a: \mathbb{R} \rightarrow \mathbb{R}$ is a function,

$* \phi:[0,+\infty) \rightarrow[0,+\infty)$ is continuous and satisfies that there exist positive numbers $l_{1}<l_{2}$ such that $l_{1} \leq \phi(x) \leq l_{2}$ for all $x \in[0,+\infty)$,

$* \lambda>0$ is a parameter, $T>0$ a constant,

$* h: \mathbb{R} \rightarrow \mathbb{R}^{+}$with $h \in X$ and $\int_{0}^{T} h(s) d s>0$,

$* f: \mathbb{R} \times[0,+\infty) \rightarrow[0,+\infty)$ satisfies that $f(\cdot, x) \in X$ and $f(t, \cdot)$ is continuous,

$* \tau: \mathbb{R} \times \mathbb{R} \rightarrow \mathbb{R}^{+}$with $\tau(\cdot, x) \in X$ and $\tau(t, \cdot)$ being continuous,

$*\left\{t_{k}\right\}$ is a real sequence satisfying that there exists $l>0$ such that $t_{k}+T=t_{k+l}$ for all $k \in \mathbb{Z}$,

$* b_{k}>-1$ constants for all $k \in \mathbb{Z}$ with $\prod_{t<t_{k} \leq t+T}\left(1+b_{k}\right)=$ constant for all $t \in \mathbb{R}$.

The purpose is to establish existence and nonexistence criterion for multiple positive $T$-periodic solutions of (1.9) with impulses effects (1.10) (system (1.9)-(1.10) for short).

The theorems obtained in this paper generalize and improve the known ones in $[2,11,14,20,39]$ and the recent publication [16].

The deduced results are different from known ones in $[5-8,12,13,17,22$, $28,30,31,33,35,36]$ when the main results are applied to (1.6), (1.7) and (1.8).

The remainder of this paper is organized as follows. In section 2, we give main results and apply them to biological models (1.6), (1.7) and (1.8), respectively. We also give an example at the end of this section. In Section 3 , the proofs of main results are presented.

\section{Main Results And Applications}

In this section, we first present the main results, then apply the main results to get multiple positive $T$-periodic solutions of equations (1.6), (1.7) and (1.8), respectively. An example is also given at the end of this section.

Choose

$X=\left\{\begin{array}{l}x: \mathbb{R} \rightarrow \mathbb{R}: x \text { is } T \text {-periodic, continuous on }\left[t_{k}, t_{k+1}\right), \\ \text { there exists the limit } \lim _{t \rightarrow t_{k}^{-}} x(t)=x\left(t_{k}^{-}\right) \text {for all } k \in \mathbb{Z} \\ \text { and } x\left(t_{k}\right)=\left(1+b_{k}\right) x\left(t_{k}^{-}\right) \text {for all } k \in \mathbb{Z}\end{array}\right\}$. 
For $x \in X$, let $\|x\|=\sup _{t \in[0 ; T]}|x(t)|$. It is easy to show that $X$ is a Banach space.

For $a \in X$, denote $a^{+}(t)=\max \{0, a(t)\}$ and $a^{-}(t)=\max \{-a(t), 0\}$. For $b_{k} \in \mathbb{R}$, denote $b_{k}^{+}=\max \left\{0, b_{k}\right\}$ and $b_{k}^{-}=\max \left\{-b_{k}, 0\right\}$.

Let us list some assumptions.

(H1) a satisfies $\exp \left(l_{1} \int_{0}^{T} a^{+}(u) d u-l_{2} \int_{0}^{T} a^{-}(u) d u\right)>\prod_{0<t_{k} \leq T}\left(1+b_{k}\right)$.

(H2) $f$ satisfies that $\max _{t \in[0, T], x \in[\sigma, 1]} f(t, x)>0$.

Denote

$$
\begin{aligned}
\sigma= & \frac{\exp \left(l_{1} \int_{0}^{T} a^{+}(u) d u-l_{2} \int_{0}^{T} a^{-}(u) d u\right)-\prod_{0<t_{k} \leq T}\left(1+b_{k}\right)}{\exp \left(l_{2} \int_{0}^{T} a^{+}(u) d u-l_{1} \int_{0}^{T} a^{-}(u) d u\right)-\prod_{0<t_{k} \leq T}\left(1+b_{k}\right)} \\
& \times \frac{\exp \left(-l_{2} \int_{0}^{T} a^{-}(u) d u\right)}{\exp \left(l_{2} \int_{0}^{T} a^{+}(u) d u\right)} \frac{\prod_{0<t_{k} \leq T}\left(1-b_{k}^{-}\right)}{\prod_{0<t_{k} \leq T}\left(1+b_{k}^{+}\right)} \\
\overline{f_{0}}= & \limsup _{x \rightarrow 0} \sup _{t \in[0, T]} \frac{f(t, x)}{x}, \quad \underline{f_{0}}=\liminf _{x \rightarrow 0} \inf _{t \in[0, T]} \frac{f(t, x)}{x}, \\
\overline{f_{\infty}}= & \limsup _{x \rightarrow+\infty} \sup _{t \in[0, T]} \frac{f(t, x)}{x}, \quad \underline{f_{\infty}}=\liminf _{x \rightarrow+\infty} \inf _{t \in[0, T]} \frac{f(t, x)}{x} .
\end{aligned}
$$

ThEOREM 2.1. Suppose that $\overline{f_{\infty}} \in[0,+\infty), f_{0} \in(0,+\infty]$ and (H1) holds. Then system (1.9)-(1.10) has at least one positive T-periodic solution if $\lambda \in$ $(A, B)$, where $A$ and $B$ are defined by

$$
\begin{gathered}
A=\frac{\exp \left(l_{2} \int_{0}^{T} a^{+}(u) d u-l_{1} \int_{0}^{T} a^{-}(u) d u\right)-\prod_{0<t_{k} \leq T}\left(1+b_{k}\right)}{\sigma \underline{f_{0}} \prod_{0<t_{k} \leq T}\left(1-b_{k}^{-}\right) \exp \left(-l_{2} \int_{0}^{T} a^{-}(u) d u\right) \int_{0}^{T} h(s) d s}, \\
B=\frac{\exp \left(l_{1} \int_{0}^{T} a^{+}(u) d u-l_{2} \int_{0}^{T} a^{-}(u) d u\right)-\prod_{0<t_{k} \leq T}\left(1+b_{k}\right)}{\overline{f_{\infty}} \prod_{0<t_{k} \leq T}\left(1+b_{k}^{+}\right) \exp \left(l_{2} \int_{0}^{T} a^{+}(u) d u\right) \int_{0}^{T} h(s) d s} .
\end{gathered}
$$

TheOrem 2.2. Suppose that $\underline{f_{\infty}} \in(0,+\infty], \overline{f_{0}} \in[0,+\infty)$ and (H1) hold. Then system (1.9)-(1.10) has at least one positive T-periodic solution if $\lambda \in$ $(A, B)$, where $A$ and $B$ are defined as follows:

$$
\begin{gathered}
A=\frac{\exp \left(l_{2} \int_{0}^{T} a^{+}(u) d u-l_{1} \int_{0}^{T} a^{-}(u) d u\right)-\prod_{0<t_{k} \leq T}\left(1+b_{k}\right)}{\sigma \underline{f_{\infty}} \prod_{0<t_{k} \leq T}\left(1-b_{k}^{-}\right) \exp \left(-l_{2} \int_{0}^{T} a^{-}(u) d u\right) \int_{0}^{T} h(s) d s}, \\
B=\frac{\exp \left(l_{1} \int_{0}^{T} a^{+}(u) d u-l_{2} \int_{0}^{T} a^{-}(u) d u\right)-\prod_{0<t_{k} \leq T}\left(1+b_{k}\right)}{\overline{f_{0}} \prod_{0<t_{k} \leq T}\left(1+b_{k}^{+}\right) \exp \left(l_{2} \int_{0}^{T} a^{+}(u) d u\right) \int_{0}^{T} h(s) d s} .
\end{gathered}
$$


TheOREM 2.3. Suppose that $\underline{f_{\infty}}=\underline{f_{0}}=+\infty$ and (H1),(H2) hold. Then there is $\lambda^{*}>0$ such that system (1.9)-(1.10) has at least two positive Tperiodic solutions for all $\lambda \in\left(0, \lambda^{*}\right)$.

TheOREM 2.4. Suppose that $\overline{f_{\infty}}=\overline{f_{0}}=0$ and (H1),(H2) hold. Then there is $\lambda^{*}>0$ such that system (1.9)-(1.10) has at least two positive Tperiodic solutions for all $\lambda>\lambda^{*}$.

TheOREM 2.5. Suppose that $\overline{f_{\infty}}<+\infty, \overline{f_{0}}<+\infty$ and (H1),(H2) hold. Then there exists $\lambda^{*}>0$ such that system (1.9)-(1.10) has no positive Tperiodic solutions for $\lambda \in\left(0, \lambda^{*}\right)$.

Theorem 2.6. Suppose that $f_{\infty}>0, \underline{f_{0}}>0$ and (H1),(H2) hold. Then there exists $\lambda^{*}>0$ such that system (1.9)-(1.10) has no positive T-periodic solutions for $\lambda \in\left(\lambda^{*},+\infty\right)$.

Now, we apply our main results to (1.6), (1.7) and (1.8), respectively, to illustrate the main results. Suppose that

(H3) $\mu, p, \tau \in X$ are $T$-periodic functions with $r(t) \geq 0, \tau(t) \geq 0, p(t) \geq 0$ for all $t \in \mathbb{R}$ and

$$
\int_{0}^{T} p(s) d s>0, \exp \left(l_{1} \int_{0}^{T} \mu^{+}(u) d u-l_{2} \int_{0}^{T} \mu^{-}(u) d u\right)>\prod_{0<t_{k} \leq T}\left(1+b_{k}\right) .
$$

Corollary 2.7. Suppose that (H3) holds. Then (1.6) has at least one positive T-periodic solution for all $\lambda>0$.

Proof. Corresponding to system (1.9)-(1.10), choose $a(t)=\mu(t), h(t)=$ $p(t)$, and $f(t, x)=e^{-r(t) x}$, replace $\tau(t, x)$ by $\tau(t)$. (H3) implies that (H1) holds. It is easy to see that

$$
\overline{f_{\infty}}=0 \in[0,+\infty), \underline{f_{0}}=+\infty \in(0,+\infty] .
$$

Hence Theorem 2.1 implies that equation (1.6) has at least one positive $T$ periodic solution for all $\lambda \in(0+\infty)$.

Corollary 2.8. Suppose that (H3) holds. Then (1.7) has at least one positive T-periodic solution for all $\lambda>0$ and $n \in[0,1) \cup(1,+\infty)$ and has at least one positive T-periodic solution for

$$
\lambda \in\left(\frac{\exp \left(l_{2} \int_{0}^{T} \mu^{+}(u) d u-l_{1} \int_{0}^{T} \mu^{-}(u) d u\right)-\prod_{0<t_{k} \leq T}\left(1+b_{k}\right)}{\sigma \prod_{0<t_{k} \leq T}\left(1-b_{k}^{-}\right) \exp \left(-l_{2} \int_{0}^{T} \mu^{-}(u) d u\right) \int_{0}^{T} p(s) d s},+\infty\right)
$$

and $n=1$. 
Proof. Corresponding to system (1.9)-(1.10), choose $a(t)=\mu(t), h(t)=$ $p(t)$, and $f(t, x)=\frac{x^{n}}{1+r(t) x^{m}}$, replace $\tau(t, x)$ by $\tau(t)$. Then (H3) implies that (H1) hold, it is easy to see that

$$
\overline{f_{\infty}}=0 \in[0,+\infty), \underline{f_{0}}=+\infty \in(0,+\infty]
$$

if $0 \leq n<1$. Hence Theorem 2.1 implies that equation (1.7) has at least one positive $T$-periodic solution for all $\lambda \in(0,+\infty)$ and $0 \leq n<1$.

One sees that $f_{\infty}=+\infty \in(0,+\infty]$ and $\overline{f_{0}}=0 \in[0,+\infty)$ if $n>1$. Hence Theorem 2.2 implies that equation (1.7) has at least one positive $T$-periodic solution for all $\lambda \in(0,+\infty)$ and $n>1$.

If $n=1$, then $\overline{f_{\infty}}=0 \in[0,+\infty)$ and $\underline{f_{0}}=1 \in(0,+\infty]$. Hence Theorem 2.1 implies that equation (1.7) has at least one positive $T$-periodic solution if $n, \lambda$ satisfy $(2.1)$.

Corollary 2.9. Suppose that (H3) holds. Then ((1.8)) has at least one positive T-periodic solution for all

$$
\lambda \in\left(\frac{\exp \left(l_{2} \int_{0}^{T} \mu^{+}(u) d u-l_{1} \int_{0}^{T} \mu^{-}(u) d u\right)-\prod_{0<t_{k} \leq T}\left(1+b_{k}\right)}{\sigma \prod_{0<t_{k} \leq T}\left(1-b_{k}^{-}\right) \exp \left(-l_{2} \int_{0}^{T} \mu^{-}(u) d u\right) \int_{0}^{T} p(s) d s},+\infty\right) .
$$

Proof. Corresponding to system (1.9)-(1.10), choose $a(t)=\mu(t), h(t)=$ $p(t)$, and $f(t, x)=x e^{-r(t) x}$, replace $\tau(t, x)$ by $\tau(t)$. Then (H3) implies that (H1) holds; it is easy to see that

$$
\overline{f_{\infty}}=0 \in[0,+\infty), \underline{f_{0}}=1 \in(0,+\infty] .
$$

Hence Theorem 2.1 implies that equation (1.8) has at least one positive $T$ periodic solution for all $\lambda$ satisfying (2.2).

EXAmple 2.10. Consider the following impulsive models

$\left\{\begin{array}{l}y^{\prime}(t)=-\left(\frac{1}{2}+\cos t\right) \frac{2+y(t)}{1+y(t)} y(t)+\lambda \frac{2-\cos ^{2} t+y^{3}(t-\tau(t))}{1+y(t-\tau(t))}, t \in \mathbb{R}, t \neq k \pi, k \in \mathbb{Z}, \\ x\left(t_{k}\right)=\frac{\sqrt{2 \pi}}{\sqrt{3}} x\left(t_{k}^{-}\right), k \in \mathbb{Z}\end{array}\right.$

where $T=2 \pi, t_{k}=k \pi+\frac{\pi}{2}, b_{k}=\frac{\sqrt{2 \pi}}{\sqrt{3}}-1, \tau: \mathbb{R} \rightarrow[0,+\infty)$ is a $2 \pi$-periodic continuous function. Corresponding to system (1.9)-(1.10), we find that

$$
\begin{aligned}
& a(t)=\frac{1}{2}+\cos t, \quad \phi(x)=\frac{2+x}{1+x} \\
& h(t) \equiv 1, \quad f(t, x)=\frac{2-\cos ^{2} t+x^{3}}{1+x}, \\
& \tau(t, x)=\tau(t), \quad b_{k}=\frac{\sqrt{2 \pi}}{\sqrt{3}}-1, t_{k}=k \pi, k \in \mathbb{Z} .
\end{aligned}
$$


It is easy to see that $l_{1}=1, l_{2}=2$. One sees that

$$
\begin{aligned}
\exp \left(l_{1} \int_{0}^{2 \pi} a^{+}(u) d u-l_{2} \int_{0}^{2 \pi} a^{-}(u) d u\right) & =\exp \left(\frac{4}{3} \pi-\sqrt{3}\right) \\
& >\prod_{0<t_{k} \leq 2 \pi}\left(1+b_{k}\right)=\frac{2 \pi}{3} .
\end{aligned}
$$

Then (H1) holds. One sees that

$$
\sigma=\frac{\exp \left(\frac{4}{3} \pi-\sqrt{3}\right)-\frac{2 \pi}{3}}{\exp \left(\frac{5}{3} \pi+\sqrt{3}\right)-\frac{2 \pi}{3}} \frac{\exp \left(\frac{2}{3} \pi-2 \sqrt{3}\right)}{\exp \left(\frac{2}{3} \pi+\sqrt{3}\right)} \frac{3}{2 \pi} .
$$

it is easy to see that $\max _{t \in[0,2 \pi], x \in[\sigma, 1]} f(t, x)>0$. Then (H2) holds. By computation, we find that

$$
\liminf _{x \rightarrow 0} \inf _{t \in[0,2 \pi]} \frac{f(t, x)}{x}=\liminf _{x \rightarrow 0} \inf _{t \in[0,2 \pi]} \frac{2-\cos ^{2} t+x^{3}}{x(1+x)}=+\infty
$$

and

$$
\liminf _{x \rightarrow+\infty} \inf _{t \in[0,2 \pi]} \frac{f(t, x)}{x}=\liminf _{x \rightarrow+\infty} \inf _{t \in[0,2 \pi]} \frac{2-\cos ^{2} t+x^{3}}{x(1+x)}=+\infty .
$$

It follows from Theorem 2.3 that there exists a constant $\lambda^{*}>0$ such that the considered system has at least two positive $2 \pi$-periodic solutions for all $\lambda \in\left(0, \lambda^{*}\right)$. In fact, one can see from the proof of Theorem 2.3 in Section 3 that $\lambda^{*}$ should be chosen in the following way

$$
\begin{aligned}
\lambda^{*} & =\frac{\exp \left(\frac{4}{3} \pi-\sqrt{3}\right)-\frac{2 \pi}{3}}{\exp \left(\frac{2}{3} \pi+\sqrt{3}\right) \frac{2 \pi}{3} 2 \pi \max _{t \in[0, T], x \in[\sigma, 1]} f(t, x)} \\
& \leq \frac{3\left(\exp \left(\frac{4}{3} \pi-\sqrt{3}\right)-\frac{2 \pi}{3}\right)}{2 \pi^{2} \exp \left(\frac{2}{3} \pi+\sqrt{3}\right)\left(1+\sigma^{3}\right)}
\end{aligned}
$$

and

$$
\lambda^{*} \geq \frac{3(1+\sigma)\left(\exp \left(\frac{4}{3} \pi-\sqrt{3}\right)-\frac{2 \pi}{3}\right)}{8 \pi^{2} \exp \left(\frac{2}{3} \pi+\sqrt{3}\right)\left(1+\sigma^{3}\right)} .
$$

REMARK 2.11. This example can not be covered by all known results. Corollaries 2.7, 2.8 and 2.9 are different from theorems obtained in [5-8,12, $13,17,22,28,30,31,33,35,36]$ even when $\phi(x) \equiv 1$.

\section{Proofs of Main Results}

In this section, we first present some background definitions in Banach spaces and state an novelty fixed point theorem. Then the main results, Theorems 2.1-2.6 are proved.

Definition 3.1. Let $X$ be a semi-ordered real Banach space. The nonempty convex closed subset $P$ of $X$ is called a cone in $X$ if ax, $x+y \in P$ for all $x, y \in P$ and $a \geq 0$ and $x \in X$ and $-x \in X$ imply $x=0$. 
Definition 3.2. Let $X$ be a Banach space. An operator $\boldsymbol{T} ; X \rightarrow X$ is completely continuous if it is continuous and maps bounded sets into precompact sets.

Lemma 3.3 (Krasnoselskii). Let $X$ be a Banach space and $P \subset X$ a cone of $X, 0 \in \Omega_{1} \subset \Omega_{2} \subset X$ open and bounded non-empty subsets. Suppose that $\boldsymbol{T}: P \cap\left(\overline{\Omega_{2}} \backslash \Omega_{1}\right) \rightarrow P$ is completely continuous. If

(i) $\|\boldsymbol{T} x\| \leq\|x\|$ for all $x \in P \partial \Omega_{1},\|\boldsymbol{T} x\| \geq\|x\|$ for all $x \in P \cap \partial \Omega_{2}$

or

(ii) $\|\boldsymbol{T} x\| \geq\|x\|$ for all $x \in P \partial \Omega_{1},\|\boldsymbol{T} x\| \leq\|x\|$ for all $x \in P \cap \partial \Omega_{2}$,

then $\boldsymbol{T}$ has at least one fixed point $x \in P \cap\left(\overline{\Omega_{2}} \backslash \Omega_{1}\right)$.

Lemma 3.4. Let $X$ be defined as in Section 2. Suppose that a, $\sigma_{1} \in X$ and (H1) holds. If $x \in X$ is a solution of the equation

$$
\left\{\begin{array}{l}
x^{\prime}(t)=-a(t) \phi(x(t)) x(t)+\sigma_{1}(t), t \neq t_{k}, k \in \mathbb{Z}, \\
x\left(t_{k}\right)=\left(1+b_{k}\right) x\left(t_{k}^{-}\right), k \in \mathbb{Z}
\end{array}\right.
$$

then

$$
x(t)=\int_{t}^{t+T} G(t, s) \sigma_{1}(s) d s, t \in \mathbb{R},
$$

where

$$
G(t, s)=\frac{\exp \left(\int_{t}^{s} \phi(x(u)) a(u) d u\right) \prod_{s<t_{k} \leq t+T}\left(1+b_{k}\right)}{\exp \left(\int_{0}^{T} \phi(x(u)) a(u) d u\right)-\prod_{0<t_{k} \leq T}\left(1+b_{k}\right)} .
$$

Proof. Since $x \in X$ is a solution of equation (3.1), we get that

$$
\left[x(t) \exp \left(\int_{0}^{t} \phi(x(u)) a(u) d u\right)\right]^{\prime}=\sigma_{1}(t) \exp \left(\int_{0}^{t} \phi(x(u)) a(u) d u\right),
$$

$t \in \mathbb{R}, t \neq t_{k}, k \in \mathbb{Z}$.

The proof is similar to the corresponding part in $[12,23]$ and is omitted.

LEMmA 3.5. Suppose that (H1) holds, $G(t, s)$ is given in (3.3). For $t \leq$ $s \leq t+T$, it holds that

$$
G(t, s) \geq \frac{\exp \left(-l_{2} \int_{0}^{T} a^{-}(u) d u\right) \prod_{0<t_{k} \leq T}\left(1-b_{k}^{-}\right)}{\exp \left(l_{2} \int_{0}^{T} a^{+}(u) d u-l_{1} \int_{0}^{T} a^{-}(u) d u\right)-\prod_{0<t_{k} \leq T}\left(1+b_{k}\right)}
$$

and

$$
G(t, s) \leq \frac{\exp \left(l_{2} \int_{0}^{T} a^{+}(u) d u\right) \prod_{0<t_{k} \leq T}\left(1+b_{k}^{+}\right)}{\exp \left(l_{1} \int_{0}^{T} a^{+}(u) d u-l_{2} \int_{0}^{T} a^{-}(u) d u\right)-\prod_{0<t_{k} \leq T}\left(1+b_{k}\right)}
$$


Proof. Let $I^{t}=[t, t+T]$, and

$$
I_{1}^{t}=\left\{t \in I^{t}: a(t) \geq 0\right\}, I_{2}^{t}=\left\{t \in I^{t}: a(t)<0\right\} .
$$

One sees from $(\mathrm{H} 1)$ that

$$
\begin{aligned}
& G(t, s) \\
& \geq \frac{\exp \left(\int_{[t, s] \cap I_{1}^{t}} \phi(x(u)) a(u) d u+\int_{[t, s] \cap I_{2}^{t}} \phi(x(u)) a(u) d u\right) \prod_{s<t_{k} \leq t+T}\left(1-b_{k}^{-}\right)}{\exp \left(\int_{0}^{T} \phi(x(u)) a(u) d u\right)-\prod_{0<t_{k} \leq T}\left(1+b_{k}\right)} \\
& \geq \frac{\exp \left(-l_{2} \int_{0}^{T} a^{-}(u) d u\right) \prod_{t<t_{k} \leq t+T}\left(1-b_{k}^{-}\right)}{\exp \left(\int_{[0, T] \cap I_{1}^{0}} \phi(x(u)) a(u) d u+\int_{[0, T] \cap I_{2}^{0}} \phi(x(u)) a(u) d u\right)-\prod_{0<t_{k} \leq T}\left(1+b_{k}\right)} \\
& \geq \frac{\exp \left(-l_{2} \int_{0}^{T} a^{-}(u) d u\right) \prod_{0<t_{k} \leq T}\left(1-b_{k}^{-}\right)}{\exp \left(l_{2} \int_{0}^{T} a^{+}(u) d u-l_{1} \int_{0}^{T} a^{-}(u) d u\right)-\prod_{0<t_{k} \leq T}\left(1+b_{k}\right)} .
\end{aligned}
$$

Then (3.5) holds. Similarly we get (3.6).

Lemma 3.6. Suppose that (H1) holds, a, $\sigma_{1} \in X, \sigma_{1}$ is nonnegative and $x \in X$ is a solution of equation (3.1). Then $x(t) \geq \sigma\|x\|$ for all $t \in \mathbb{R}$, where $\sigma$ is defined in Section 2.

Proof. Since (H1) holds, we get from Lemma 3.4 that (3.2) holds. Then Lemma 3.5 implies that

$$
\begin{aligned}
& x(t) \\
& \geq \frac{\exp \left(-l_{2} \int_{0}^{T} a^{-}(u) d u\right) \prod_{0<t_{k} \leq T}\left(1-b_{k}^{-}\right)}{\exp \left(l_{2} \int_{0}^{T} a^{+}(u) d u-l_{1} \int_{0}^{T} a^{-}(u) d u\right)-\prod_{0<t_{k} \leq T}\left(1+b_{k}\right)} \int_{t}^{t+T} \sigma_{1}(s) d s \\
& =\frac{\exp \left(-l_{2} \int_{0}^{T} a^{-}(u) d u\right) \prod_{0<t_{k} \leq T}\left(1-b_{k}^{-}\right)}{\exp \left(l_{2} \int_{0}^{T} a^{+}(u) d u-l_{1} \int_{0}^{T} a^{-}(u) d u\right)-\prod_{0<t_{k} \leq T}\left(1+b_{k}\right)} \int_{0}^{T} \sigma_{1}(s) d s,
\end{aligned}
$$

and similarly

$$
x(t) \leq \frac{\exp \left(l_{2} \int_{0}^{T} a^{+}(u) d u\right) \prod_{0<t_{k} \leq T}\left(1+b_{k}^{+}\right)}{\exp \left(l_{1} \int_{0}^{T} a^{+}(u) d u-l_{2} \int_{0}^{T} a^{-}(u) d u\right)-\prod_{0<t_{k} \leq T}\left(1+b_{k}\right)} \int_{0}^{T} \sigma_{1}(s) d s .
$$

Then

$$
\|x\| \leq \frac{\exp \left(l_{2} \int_{0}^{T} a^{+}(u) d u\right) \prod_{0<t_{k} \leq T}\left(1+b_{k}^{+}\right)}{\exp \left(l_{1} \int_{0}^{T} a^{+}(u) d u-l_{2} \int_{0}^{T} a^{-}(u) d u\right)-\prod_{0<t_{k} \leq T}\left(1+b_{k}\right)} \int_{0}^{T} \sigma_{1}(s) d s .
$$


It follows that

$$
\begin{aligned}
x(t) \geq & \frac{\exp \left(l_{1} \int_{0}^{T} a^{+}(u) d u-l_{2} \int_{0}^{T} a^{-}(u) d u\right)-\prod_{0<t_{k} \leq T}\left(1+b_{k}\right)}{\exp \left(l_{2} \int_{0}^{T} a^{+}(u) d u-l_{1} \int_{0}^{T} a^{-}(u) d u\right)-\prod_{0<t_{k} \leq T}\left(1+b_{k}\right)} \times \\
& \frac{\exp \left(-l_{2} \int_{0}^{T} a^{-}(u) d u\right)}{\prod_{0<t_{k} \leq T}\left(1-b_{k}^{-}\right)}\|x\| \\
= & \sigma\|x\|, \quad t \in \mathbb{R} .
\end{aligned}
$$

Choose

$$
\begin{gathered}
P=\left\{x \in X: x\left(t_{k}\right)=\left(1+b_{k}\right) x\left(t_{k}^{-}\right) \text {for all } k \in \mathbb{Z}\right. \\
\text { and } x(t) \geq \sigma\|x\| \text { for all } t \in \mathbb{R}\} .
\end{gathered}
$$

It is easy to see that $P$ is a cone in the space $X$ defined in Section 2. Define the operator $\mathbf{T}: X \rightarrow X$ by

$$
\mathbf{T} x(t)=\lambda \int_{t}^{t+T} G(t, s) h(s) f(s, x(s-\tau(s, x(s)))) d s, t \in \mathbb{R}
$$

for $x \in X$, where $G(t, s)$ is defined by $(3.3)$.

Lemma 3.7. Suppose that (H1) holds. Then

(i) it holds that

$(\boldsymbol{T} x)^{\prime}(t)=-a(t) \phi(x(t))(\boldsymbol{T} x)(t)+\lambda h(t) f(t, x(t-\tau(t, x(t)))), t \in \mathbb{R}, t \neq t_{k}, k \in \mathbb{Z}$

and

$$
(\boldsymbol{T} x)\left(t_{k}\right)=\left(1+b_{k}\right)(\boldsymbol{T} x)\left(t_{k}^{-}\right), k \in \mathbb{Z}
$$

(ii) for $x \in P,(\boldsymbol{T} x)(t) \geq \sigma\|\boldsymbol{T} x\|$ for all $t \in \mathbb{R}$ and $x \in P$, i.e., $\boldsymbol{T} P \subseteq P$;

(iii) $\boldsymbol{T}$ is completely continuous on $P$;

(iv) $x \in X$ is a positive $T$-periodic solution of system (1.9)-(1.10) if and only if $x$ is a fixed point of operator $\boldsymbol{T}$ in $P$.

Proof. The proofs are simple, standard and are omitted.

Proof of Theorem 2.1. Let $\lambda \in(A, B)$ be fixed. From $\lambda<B$ defined in Theorem 2.1, we choose $\epsilon>0$ and $R_{2}>0$ such that

$$
\lambda\left(\overline{f_{\infty}}+\epsilon\right) \frac{\prod_{0<t_{k} \leq T}\left(1+b_{k}^{+}\right) \exp \left(l_{2} \int_{0}^{T} a^{+}(u) d u\right) \int_{0}^{T} h(s) d s}{\exp \left(l_{1} \int_{0}^{T} a^{+}(u) d u-l_{2} \int_{0}^{T} a^{-}(u) d u\right)-\prod_{0<t_{k} \leq T}\left(1+b_{k}\right)} \leq 1
$$

and

$$
\frac{f(t, x)}{x} \leq \overline{f_{\infty}}+\epsilon, \quad t \in[0, T], x \geq R_{2}
$$


SteP 1. Set $\Omega_{1}=\left\{x \in X:\|x\|<R_{2} / \sigma\right\}$. If $y \in P \cap \partial \Omega_{1}$, then $y(t) \geq$ $\sigma\|y\|=R_{2}$ and

$$
\begin{aligned}
& \mathbf{T} y(t) \\
& =\lambda \int_{t}^{t+T} G(t, s) h(s) f(s, y(s-\tau(s, y(s)))) d s \\
& \leq \lambda\left(\overline{f_{\infty}}+\epsilon\right) \int_{t}^{t+T} G(t, s) h(s) y(s, \tau(s, y(s))) d s \\
& \leq \lambda\left(\overline{f_{\infty}}+\epsilon\right)\|y\| \int_{t}^{t+T} G(t, s) h(s) d s \\
& \leq \lambda\left(\overline{f_{\infty}}+\epsilon\right) \frac{\|y\| \prod_{0<t_{k} \leq T}\left(1+b_{k}^{+}\right) \exp \left(l_{2} \int_{0}^{T} a^{+}(u) d u\right) \int_{0}^{T} h(s) d s}{\exp \left(l_{1} \int_{0}^{T} a^{+}(u) d u-l_{2} \int_{0}^{T} a^{-}(u) d u\right)-\prod_{0<t_{k} \leq T}\left(1+b_{k}\right)} \\
& \leq\|y\| .
\end{aligned}
$$

Then $\|\mathbf{T} y\| \leq\|y\|$ for all $y \in P \cap \partial \Omega_{1}$.

SteP 2. Since $\lambda>A$ defined in Theorem 2.1, choose $\epsilon>0$ such that

$$
\lambda \sigma\left(\underline{f_{0}}-\epsilon\right) \frac{\prod_{0<t_{k} \leq T}\left(1-b_{k}^{-}\right) \exp \left(-l_{2} \int_{0}^{T} a^{-}(u) d u\right) \int_{0}^{T} h(s) d s}{\exp \left(l_{2} \int_{0}^{T} a^{+}(u) d u-l_{1} \int_{0}^{T} a^{-}(u) d u\right)-\prod_{0<t_{k} \leq T}\left(1+b_{k}\right)} \geq 1 .
$$

Choose $R_{1}$ sufficiently small such that $0<R_{1}<R_{2}$ and

$$
\frac{f(t, x)}{x}>\underline{f_{0}}-\epsilon, t \in[0, T], x \in\left[0, R_{1}\right] .
$$

Let $\Omega_{2}=\left\{x \in X:\|x\|<R_{1}\right\}$. For $y \in P \cap \partial \Omega_{2}$, we find $0 \leq y(t) \leq\|y\|=R_{1}$, and

$$
\begin{aligned}
\mathbf{T} y(t) & \geq \lambda\left(\underline{f_{0}}-\epsilon\right) \int_{t}^{t+T} G(t, s) h(s) y(s-\tau(s, y(s))) d s \\
& \geq \lambda\left(\underline{f_{0}}-\epsilon\right) \int_{t}^{t+T} G(t, s) h(s) \sigma\|y\| d s \\
& \geq \lambda \sigma\left(\underline{f_{0}}-\epsilon\right) \frac{\|y\| \prod_{0<t_{k} \leq T}\left(1-b_{k}^{-}\right) \exp \left(-l_{2} \int_{0}^{T} a^{-}(u) d u\right) \int_{0}^{T} h(s) d s}{\exp \left(l_{2} \int_{0}^{T} a^{+}(u) d u-l_{1} \int_{0}^{T} a^{-}(u) d u\right)-\prod_{0<t_{k} \leq T}\left(1+b_{k}\right)} \\
& \geq\|y\| .
\end{aligned}
$$

Then $\|\mathbf{T} y\| \geq\|y\|$ for all $y \in P \cap \partial \Omega_{2}$. Hence Lemma 3.7 and 3.3 imply that $\mathbf{T}$ has at least one fixed point $y$ such that $R_{1} \leq\|y\| \leq R_{2} / \sigma$ that is a positive $T$-periodic solution of system (1.9)-(1.10).

Proof of Theorem 2.2. The proof is similar to that of Theorem 2.1 and it is omitted. 
Proof of Theorem 2.3. Choose

$$
\lambda^{*}=\frac{\exp \left(l_{1} \int_{0}^{T} a^{+}(u) d u-l_{2} \int_{0}^{T} a^{-}(u) d u\right)-\prod_{0<t_{k} \leq T}\left(1+b_{k}\right)}{\exp \left(l_{2} \int_{0}^{T} a^{+}(u) d u\right) \prod_{0<t_{k} \leq T}\left(1+b_{k}^{+}\right) \int_{0}^{T} h(s) d s \max _{t \in[0, T], x \in[\sigma, 1]} f(t, x)} .
$$

For $\lambda \in\left(0, \lambda^{*}\right)$, we prove that system (1.9)-(1.10) has at least two positive $T$-periodic solutions.

Choose $\Omega_{0}=\{x \in X:\|x\|<1\}$. Then $x \in P \cap \partial \Omega_{0}$ implies that $\|x\| \in[\sigma, 1]$, we have

$$
\begin{aligned}
& \mathbf{T} x(t) \\
& =\lambda \int_{t}^{t+T} G(t, s) h(s) f(s, x(s-\tau(s, x(s)))) d s \\
& \leq \lambda^{*} \frac{\exp \left(l_{2} \int_{0}^{T} a^{+}(u) d u\right) \prod_{0<t_{k} \leq T}\left(1+b_{k}^{+}\right) \int_{t}^{t+T} h(s) f(s, x(s-\tau(s, x(s)))) d s}{\exp \left(l_{1} \int_{0}^{T} a^{+}(u) d u-l_{2} \int_{0}^{T} a^{-}(u) d u\right)-\prod_{0<t_{k} \leq T}\left(1+b_{k}\right)} \\
& =\lambda^{*} \frac{\exp \left(l_{2} \int_{0}^{T} a^{+}(u) d u\right) \prod_{0<t_{k} \leq T}\left(1+b_{k}^{+}\right) \int_{0}^{T} h(s) d s \max _{t \in[0, T], x \in[\sigma, 1]} f(t, x)}{\exp \left(l_{1} \int_{0}^{T} a^{+}(u) d u-l_{2} \int_{0}^{T} a^{-}(u) d u\right)-\prod_{0<t_{k} \leq T}\left(1+b_{k}\right)} \\
& \leq 1=\|x\| .
\end{aligned}
$$

We get $\|\mathbf{T} x\| \leq\|x\|$ for $x \in P \cap \partial \Omega_{0}$.

Choose $G_{0}>0$ such that

$$
\lambda \frac{G_{0} \sigma \exp \left(l_{2} \int_{0}^{T} a^{+}(u) d u\right) \prod_{0<t_{k} \leq T}\left(1+b_{k}^{+}\right) \int_{0}^{T} h(s) d s}{\exp \left(l_{1} \int_{0}^{T} a^{+}(u) d u-l_{2} \int_{0}^{T} a^{-}(u) d u\right)-\prod_{0<t_{k} \leq T}\left(1+b_{k}\right)} \geq 1 .
$$

Since

$$
\liminf _{x \rightarrow+\infty} \inf _{t \in[0, T]} \frac{f(t, x)}{x}=\underline{f_{\infty}}=+\infty, \liminf _{x \rightarrow 0} \inf _{t \in[0, T]} \frac{f(t, x)}{x}=\underline{f_{0}}=+\infty,
$$

we get that there exist constants $0<R_{1}<\sigma<1<\sigma R_{2}$ such that

$$
\frac{f(t, x)}{x} \geq G_{0}, t \in[0, T], x \geq R_{2}
$$

and

$$
\frac{f(t, x)}{x} \geq G_{0}, t \in[0, T], x \leq R_{1}
$$


Let $\Omega_{1}=\left\{x \in X:\|x\|<R_{1}\right\}$ and $\Omega_{2}=\left\{x \in X:\|x\|<R_{2} / \sigma\right\}$. Then, for $x \in P \cap \partial \Omega_{2}$, one has $x(t) \geq \sigma\|x\|=\sigma \frac{R_{2}}{\sigma}=R_{2}$ and

$\mathbf{T} x(t)$

$$
\begin{aligned}
& =\lambda \int_{t}^{t+T} G(t, s) h(s) f(s, x(s-\tau(s, x(s)))) d s \\
& \geq \lambda \frac{G_{0} \exp \left(-l_{2} \int_{0}^{T} a^{-}(u) d u\right) \prod_{0<t_{k} \leq T}\left(1-b_{k}^{-}\right) \int_{t}^{t+T} h(s) x(s-\tau(s, x(s))) d s}{\exp \left(l_{2} \int_{0}^{T} a^{+}(u) d u-l_{1} \int_{0}^{T} a^{-}(u) d u\right)-\prod_{0<t_{k} \leq T}\left(1+b_{k}\right)} \\
& \geq \lambda \frac{G_{0} \sigma\|x\| \exp \left(l_{2} \int_{0}^{T} a^{+}(u) d u\right) \prod_{0<t_{k} \leq T}\left(1+b_{k}^{+}\right) \int_{0}^{T} h(s) d s}{\exp \left(l_{1} \int_{0}^{T} a^{+}(u) d u-l_{2} \int_{0}^{T} a^{-}(u) d u\right)-\prod_{0<t_{k} \leq T}\left(1+b_{k}\right)} \\
& \geq\|x\| .
\end{aligned}
$$

We get $\|\mathbf{T} x\| \geq\|x\|$ for $x \in P \cap \partial \Omega_{2}$.

$$
\text { For } x \in P \cap \partial \Omega_{1} \text {, one has } 0 \leq x(t) \leq\|x\|=R_{1} \text {, then }
$$

$\mathbf{T} x(t)$

$$
\begin{aligned}
& =\lambda \int_{t}^{t+T} G(t, s) h(s) f(s, x(s-\tau(s, x(s)))) d s \\
& \geq \lambda \frac{G_{0} \exp \left(-l_{2} \int_{0}^{T} a^{-}(u) d u\right) \prod_{0<t_{k} \leq T}\left(1-b_{k}^{-}\right) \int_{t}^{t+T} h(s) x(s-\tau(s, x(s))) d s}{\exp \left(l_{2} \int_{0}^{T} a^{+}(u) d u-l_{1} \int_{0}^{T} a^{-}(u) d u\right)-\prod_{0<t_{k} \leq T}\left(1+b_{k}\right)} \\
& \geq \lambda \frac{G_{0} \sigma\|x\| \exp \left(l_{2} \int_{0}^{T} a^{+}(u) d u\right) \prod_{0<t_{k} \leq T}\left(1+b_{k}^{+}\right) \int_{0}^{T} h(s) d s}{\exp \left(l_{1} \int_{0}^{T} a^{+}(u) d u-l_{2} \int_{0}^{T} a^{-}(u) d u\right)-\prod_{0<t_{k} \leq T}\left(1+b_{k}\right)} \\
& \geq\|x\| .
\end{aligned}
$$

We get $\|\mathbf{T} x\| \geq\|x\|$ for $x \in P \cap \partial \Omega_{1}$. From above discussion, applying Lemma 3.7 and Lemma 3.3, we get that $\mathbf{T}$ has at least two fixed point $y_{1}, y_{2}$ such that $R_{1} \leq\left\|y_{1}\right\| \leq R_{1}<\sigma<1 \leq R_{2} \leq\left\|y_{2}\right\| \leq R_{2} / \sigma$. Hence $y_{1}, y_{2}$ are two positive $T$-periodic solution of system (1.9)-(1.10).

\section{Proof of Theorem 2.4. Choose}

$$
\lambda^{*}=\frac{\exp \left(l_{2} \int_{0}^{T} a^{+}(u) d u-l_{1} \int_{0}^{T} a^{-}(u) d u\right)-\prod_{0<t_{k} \leq T}\left(1+b_{k}\right)}{\sigma \exp \left(-l_{2} \int_{0}^{T} a^{-}(u) d u\right) \prod_{0<t_{k} \leq T}\left(1-b_{k}^{-}\right) \int_{0}^{T} h(s) d s \min _{t \in[0, T], x \in[\sigma, 1]} f(t, x)} .
$$

The remainder of the proof is similar to that of Theorem 2.3 and it is omitted. 
Proof of Theorem 2.5. Since $\max _{t \in[0, T], x \in[\sigma, 1]} f(t, x)>0$ and

$$
\limsup _{x \rightarrow+\infty} \sup _{t \in[0, T]} \frac{f(t, x)}{x}=\overline{f_{\infty}}<+\infty, \limsup _{x \rightarrow 0} \sup _{t \in[0, T]} \frac{f(t, x)}{x}=\overline{f_{0}}<+\infty,
$$

we get that there exist positive numbers $\epsilon_{1}, \epsilon_{2}$ and $r_{1}<\sigma<1<r_{2}$ such that

$$
\frac{f(t, x)}{x} \leq\left(\overline{f_{\infty}}+\epsilon_{1}\right), t \in[0, T], x \in\left[0, r_{1}\right]
$$

and

$$
\frac{f(t, x)}{x} \leq\left(\overline{f_{0}}+\epsilon_{2}\right), t \in[0, T], x \geq r_{2}
$$

Denote

$$
A=\max \left\{\overline{f_{\infty}}+\epsilon_{1}, \overline{f_{0}}+\epsilon_{2}, \max _{t \in[0, T], x \in\left[r_{1}, r_{2}\right]} \frac{f(t, x)}{x}\right\} .
$$

It follows from $\max _{t \in[0, T], x \in[\sigma, 1]} f(t, x)>0$ that $A>0$ and $f(t, x) \leq A x$ for all $t \in[0, T]$ and $x \in[0,+\infty)$. Choose

$$
\lambda^{*}=\frac{\exp \left(l_{1} \int_{0}^{T} a^{+}(u) d u-l_{2} \int_{0}^{T} a^{-}(u) d u\right)-\prod_{0<t_{k} \leq T}\left(1+b_{k}\right)}{A \exp \left(l_{2} \int_{0}^{T} a^{+}(u) d u\right) \prod_{0<t_{k} \leq T}\left(1+b_{k}^{+}\right) \int_{0}^{T} h(s) d s} .
$$

For $\lambda \in\left(0, \lambda^{*}\right)$, if system (1.9)-(1.10) has a positive $T$-periodic solution $x$, then

$$
\begin{aligned}
& x(t)=\mathbf{T} x(t) \\
& =\lambda \int_{t}^{t+T} G(t, s) h(s) f(s, x(s-\tau(s, x(s)))) d s \\
& \leq \lambda \frac{A \exp \left(l_{2} \int_{0}^{T} a^{+}(u) d u\right) \prod_{0<t_{k} \leq T}\left(1+b_{k}^{+}\right) \int_{t}^{t+T} h(s) x(s-\tau(s, x(s))) d s}{\exp \left(l_{1} \int_{0}^{T} a^{+}(u) d u-l_{2} \int_{0}^{T} a^{-}(u) d u\right)-\prod_{0<t_{k} \leq T}\left(1+b_{k}\right)} \\
& \leq \lambda \frac{A\|x\| \exp \left(l_{2} \int_{0}^{T} a^{+}(u) d u\right) \prod_{0<t_{k} \leq T}\left(1+b_{k}^{+}\right) \int_{0}^{T} h(s) d s}{\exp \left(l_{1} \int_{0}^{T} a^{+}(u) d u-l_{2} \int_{0}^{T} a^{-}(u) d u\right)-\prod_{0<t_{k} \leq T}\left(1+b_{k}\right)} .
\end{aligned}
$$

Then

$$
\begin{aligned}
\|x\| & \leq \lambda \frac{A\|x\| \exp \left(l_{2} \int_{0}^{T} a^{+}(u) d u\right) \prod_{0<t_{k} \leq T}\left(1+b_{k}^{+}\right) \int_{0}^{T} h(s) d s}{\exp \left(l_{1} \int_{0}^{T} a^{+}(u) d u-l_{2} \int_{0}^{T} a^{-}(u) d u\right)-\prod_{0<t_{k} \leq T}\left(1+b_{k}\right)} \\
& <\lambda^{*} \frac{A\|x\| \exp \left(l_{2} \int_{0}^{T} a^{+}(u) d u\right) \prod_{0<t_{k} \leq T}\left(1+b_{k}^{+}\right) \int_{0}^{T} h(s) d s}{\exp \left(l_{1} \int_{0}^{T} a^{+}(u) d u-l_{2} \int_{0}^{T} a^{-}(u) d u\right)-\prod_{0<t_{k} \leq T}\left(1+b_{k}\right)} \\
& =\|x\|,
\end{aligned}
$$


which is a contradiction. Hence For $\lambda \in\left(0, \lambda^{*}\right)$, system (1.9)-(1.10) has no positive $T$-periodic solution. The proof is completed.

Proof of Theorem 2.6. Since $\max _{t \in[0, T], x \in[\sigma, 1]} f(t, x)>0$ and

$$
\liminf _{x \rightarrow+\infty} \inf _{t \in[0, T]} \frac{f(t, x)}{x}=\underline{f_{\infty}}>0, \liminf _{x \rightarrow 0} \inf _{t \in[0, T]} \frac{f(t, x)}{x}=\underline{f_{0}}>0,
$$

we get that there exist positive numbers $\epsilon_{1}<\underline{f_{\infty}}, \epsilon_{2}<\underline{f_{0}}$ and $r_{1}<\sigma<1<r_{2}$ such that

$$
\left.\frac{f(t, x)}{x} \geq \underline{\left(f_{\infty}\right.}-\epsilon_{1}\right), t \in[0, T], x \in\left[0, r_{1}\right]
$$

and

Denote

$$
\frac{f(t, x)}{x} \geq\left(\underline{f_{0}}-\epsilon_{2}\right), t \in[0, T], x \geq r_{2} .
$$

Choose

$$
B=\min \left\{\underline{f_{\infty}}-\epsilon_{1}, \underline{f_{0}}-\epsilon_{2}, \min _{t \in[0, T], x \in\left[r_{1}, r_{2}\right]} \frac{f(t, x)}{x}\right\} .
$$

$$
\lambda^{*}=\frac{\exp \left(l_{2} \int_{0}^{T} a^{+}(u) d u-l_{2} \int_{0}^{T} a^{-}(u) d u\right)-\prod_{0<t_{k} \leq T}\left(1+b_{k}\right)}{B \sigma \exp \left(-l_{2} \int_{0}^{T} a^{-}(u) d u\right) \prod_{0<t_{k} \leq T}\left(1-b_{k}^{-}\right) \int_{0}^{T} h(s) d s} .
$$

The remainder of the proof is similar to that of Theorem 2.5 and it is omitted.

\section{ACKNOWLEDGEMENTS.}

The author expresses his thanks to the referees for their valuable suggestions and to the editors for their careful work.

\section{REFERENCES}

[1] B. Ahmad and J. J. Nieto, Existence and approximation of solutions for a class of nonlinear impulsive functional differential equations with anti-periodic boundary conditions, Nonlinear Anal. 69 (2008), 3291-3298.

[2] S. Cheng and G. Zhang, Existence of positive periodic solutions for non-autonomous functional differential equations, Electronic J. Differential Equations 59 (2001), 1-8.

[3] J. Chu, Impulsive periodic solutions of first-order singular differential equations, Bull. Lond. Math. Soc. 40 (2008), 143-150.

[4] P. Georgescu, H. Zhang and L. Chen, Bifurcation of nontrivial periodic solutions for an impulsively controlled pest management model, Appl. Math. Comput. 202 (2008), 675-687.

[5] W. Gurney, S. Blythe and R. Nisbet, Nicholson's Blowflies revised, Nature 287 (1980), 17-21.

[6] J. Hale and S. Lunel, Introduction to functional differential equations, New York, Berlin, Springer-Verlag, 1993.

[7] Y. Kuang and H. Smith, Slowly oscillating periodic solutions of nonautonomous statedependent delay equations, Nonlinear Anal. 19 (1992), 855-872.

[8] V. Lakshmikantham, D. Bainov and P. Simeonov, Theory of impulsive differential equations. Singapore, World Scientist, 1989. 
[9] E. K. Lee and Y-H. Lee, Multiple positive solutions of singular two point boundary value problems for second order impulsive differential equations, Appl. Math. Comput., 158 (2004), 745-759.

[10] J. Li, J. J. Nieto and J. Shen, Impulsive periodic boundary value problems of firstorder differential equations, J. Math. Anal. Appl. 325 (2007), 226-236.

[11] J. Li and J. Shen, Existence of positive periodic solutions to a class of functional differential equations with impulses, Math. Appl. (Wuhan) 17 (2004), 456-463.

[12] W. Li and H. Huo. Global attractivity of positive periodic solutions for an impulsive delay periodic model of respiratory dynamics, J. Comput. Appl. Math., 174 (2005), $227-238$.

[13] W. Li and Z. Wang, Existence and global attractivity of positive periodic solutions of a survival model of red blood cells, Comput. Appl. Math. 50 (2005), 41-47.

[14] X. Li, X. Lin, D. Jiang and X. Zhang, Existence and multiplicity of positive periodic solutions to functional differential equations with impulse effects, Nonlinear Anal., 62 (2005), 683-701.

[15] X. Li, X. Zhang and D. Jiang, A new existence theory for positive periodic solutions to functional differential equations with impulse effects, Comput. Math. Appl. 51 (2006), 1761-1772.

[16] Y. Li, X. Fan and L. Zhao, Positive periodic solutions of functional differential equations with impulses and a parameter, Comput. Math. Appl. 56 (2008), 25562560.

[17] G. Liu, J. Yan and F. Zhang, Existence and global attractivity of unique positive periodic solution for a model of hematopoiesis, J. Math. Anal. Appl. 334 (2007), $157-171$.

[18] S. Liu, Y. Pei, C. Li and L. Chen, Three kinds of TVS in a SIR epidemic model with saturated infectious force and vertical transmission. Appl. Math. Model. 33 (2009), 1923-1932.

[19] Y. Liu, Positive solutions of periodic boundary value problems for nonlinear firstorder impulsive differential equations, Nonlinear Anal. 70 (2009) , 2106-2122.

[20] Y. Liu and W. Ge, Positive periodic solutions of state-dependent functional differential equations, Appl. Anal. 84 (2005), 1079-1094.

[21] Y. Liu, J. Xia and W. Ge, Positive periodic solutions of impulsive functional differential equations, J. Appl. Math. Comput. 19 (2005), 261-280.

[22] Y. Liu and B. Zhang, Global attractivity of a class of delay differential equations with impulses, ANZIAM J. 45 (2003), 271-284.

[23] Z. Luo and Z. Jing, Periodic boundary value problem for first-order impulsive functional differential equations. Comput. Math. Appl. 55 (2008), 2094-2107.

[24] Z. Luo and J. J. Nieto, New results for the periodic boundary value problem for impulsive integro-differential equations. Nonlinear Anal. 70 (2009), 2248-2260

[25] L. Nie, Z. Teng, L. Hu and J. Peng, Existence and stability of periodic solution of a Lotka-Volterra predator-prey model with state dependent impulsive effects, J. Comput. Appl. Math. 224 (2009), 544-555.

[26] J. J. Nieto and R. Rodriguez-Lopez, Comparison results and approximation of solutions for impulsive functional differential equations, Dyn. Contin. Discrete Impuls. Syst. Ser. A Math. Anal. 15 (2008), 169-215.

[27] J. J. Nieto and R. Rodriguez-Lopez, Boundary value problems for a class of impulsive functional equations, Comput. Math. Appl. 55 (2008), 2715-2731.

[28] E. Pielou, Mathematics ecology, New York, Wiley-Inter-science, 1977.

[29] D. Qian and X. Li, Periodic solutions for ordinary differential equations with sublinear impulsive effects, J. Math. Anal. Appl. 303 (2005), 288-303. 
[30] G. Rost, On the global attractivity controversy for a delay model of hematopoiesis, Appl. Math. Comput. 190 (2007), 846-850.

[31] S. Saker and S. Agarwal, Oscillatory and global attractivity in a periodic Nicholson's blowflies model, Math. Comput. Modelling 35 (2002), 719-731.

[32] J. Shen and J. Li, Existence and global attractivity of positive periodic solutions for impulsive predator-prey model with dispersion and time delays, Nonlinear Anal. Real World Appl. 10 (2009), 227-243.

[33] S. Tang and L. Chen, Global attractivity in a "food-limited" population model with impulsive effects, J. Math. Anal. Appl. 292 (2004), 211-221.

[34] H. Wang, Positive periodic solutions of functional differential equations, J. Differential Equations 202 (2004), 354-366.

[35] M. Wazewska-Czyzewska and A. Lasota, Mathematical problems of the dynamics of a system of red blood cells, Mat. Stos. (3) 6 (1976), 23-40.

[36] P. Weng and M. Liang, The existence and behavior of periodic solutions of a Hematopoiesis model, Math. Appl. (Wuhan) 8 (1995), 434-439.

[37] J. Yan, Existence of positive periodic solutions of impulsive functional differential equations with two parameters, J. Math. Anal. Appl. 327 (2007), 854-868.

[38] J. Yan, A. Zhao and J. J. Nieto, Existence and global attractivity of positive periodic solution of periodic single-species impulsive Lotka-Volterra systems, Math. Comput. Model. 40 (2004), 509-518.

[39] G. Zhang and S. Cheng, Positive periodic solutions of nonautonomous functional differential equations depend on a parameter, Abstract Anal. Appl. 7 (2002), 279286.

[40] N. Zhang, B. Dai and X. Qian, Periodic solutions for a class of higher-dimension functional differential equations with impulses, Nonlinear Anal. 68 (2008), 629-638.

[41] X. Zhang, X. Li, D. Jiang and K. Wang, Multiplicity Positive Solutions to Periodic Problems for First-Order Impulsive Differential Equations, Comput. Math. Appl. 52 (2006), 953-966.

[42] X. Zhang, J. Yan and A. Zhao, Existence of positive periodic solutions for an impulsive differential equation, Nonlinear Anal. 68 (2008), 3209-3216.

Y. Liu

Department of Mathematics

Guangdong University of Business Studies

Guangzhou 510320

P. R. China

E-mail: liuyuji888@sohu.com

Received: 14.11.2009. 